\title{
NEUTRON DOSIMETRY WITH NUCLEAR EMULSIONS
}

\author{
M. Höfert \\ CERN, Geneva, Switzerland \\ and \\ E. Piesch \\ Karlsruhe Nuclear Research Centre, Karlsruhe, Fed. Rep. Germany
}




\section{INTRODUCTION}

The dosimetry of neutrons ranging in energy over several orders of magnitude from thermal to relativistic energies is a challenging problem. One of the earliest methods employed in personnel neutron dosimetry is the use of special photographic emulsions where, after development and magnification in the microscope, nuclear tracks can be observed. Over the last years it seemed that this method would be rapidly replaced by modern track etch detectors or albedo neutron dosemeters, these being superior to the nuclear emulsion in most of the dosimetric properties. However, so far the advances in the above techniques have not justified a changeover in all cases, but have rather stimulated new efforts to improve the neutron detection capabilities of nuclear emulsions.

The history and whole field of neutron dosimetry with nuclear emulsion has already been discussed in earlier publications. The reader is therefore referred to review articles on dosimetry for more references about all aspects of these techniques [1-3]. This paper therefore discusses mainly recent results found with the nuclear film type A (NTA) produced by Kodak, the most commonly used personnel neutron monitoring film.

\section{NEUTRON DETECTION IN NUCLEAR EMULSIONS}

The most important neutron reaction that will lead to tracks of recoil protons in nuclear emulsions is the elastic scattering with hydrogen nuclei which takes place in the emulsion itself, in the plastic base, in the packaging of the emulsion, in the film holder and, for high neutron energies, in the body of the dosemeter wearer.

Monoenergetic neutrons produce recoil protons in the emulsion with a uniform distribution in energy ranging from zero to the primary energy of the incident neutrons.

There exists, however, a threshold for the detection of low-energy recoil protons. It is commonly assumed that protons of $0.5 \mathrm{MeV}$ lead to tracks of not more than three to four grains, which could easily be missed in an observation because of background grains and/or fading effects. Otherwise, neutron-induced recoil proton tracks are registered by the NTA emulsion in the energy range from less than $1 \mathrm{MeV}$ up to the highest available energies.

At neutron energies above $10 \mathrm{MeV}$, however, recoil proton tracks become rather long $(1 \mathrm{~mm}$ at $14 \mathrm{MeV}$ ), and their structure is rather faint owing to the small stopping power involved. For neutron incidence parallel with the emulsion, tracks will cross the field size used in the microscope for track counting, and track evaluation becomes difficult.

Additional problems with respect to possible neutron spectroscopy are the finite thickness of the emulsion which does not allow the full lengths of the tracks to be observed, except for the perpendicular incidence of the neutrons, and the projection of the microscope tracks into the plane of the emulsion. Thus generally only track densities are counted.

A further interaction to be considered is the ${ }^{14} \mathrm{~N}(\mathrm{n}, \mathrm{p}){ }^{14} \mathrm{C}$ reaction with a high cross-section for thermal and epithermal neutrons emitting a $0.6 \mathrm{MeV}$ proton. This reaction is, however, mostly of academic interest because of the short track length at the limit of detection [4].

Finally, the reaction ${ }^{12} \mathrm{C}(\mathrm{n}, \mathrm{n}) 3 \boldsymbol{\alpha}$ may play a role in the formation of tracks in a nuclear emulsion. Above threshold energies of about $12 \mathrm{MeV}$, spallation reaction will lead to multiple-prong stars which look spectacular in the microscope, especially when a silver atom is hit. Stars are also observed in non-irradiated emulsions as background from cosmic radiation. Hence the dosimetric 
information becomes doubtful as, on the average, even in cases of exposures outside the shield of high-energy proton accelerators, only one of these events is observed for about 50 normal proton recoil tracks.

Experiments to improve the response of nuclear emulsions either by mixing into them elements with high cross-sections for neutron reactions or by exposing them when surrounded by special radiators, have not led to any routine application $[5,6]$.

\section{DOSIMETRIC PROPERTIES}

\subsection{Energy dependence}

Calculations and experiments have shown that the response of the NTA film, i.e. the number of tracks per unit area and dose equivalent depends on the neutron energy. Above a threshold energy of about $1 \mathrm{MeV}$, the number of recoil proton tracks increases with energy up to $10 \mathrm{MeV}$ (Fig. 1) and drops at higher energies. By using special radiators of hydrogenous material or placing the NTA film package in a plastic film holder, the energy range can be extended to higher neutron energies [6]. When considering the actual response in stray neutron fields, it should be noted that spectral distributions rather than monoenergetic neutrons are encountered. In cases where the neutron spectrum in an installation varies only negligibly, from place to place, the mean value of response factors can be used, which has been found by calibrating the NTA film in its badge in the neutron field of interest.

It should be mentioned here that the NTA film is still frequently used as a personnel monitor in neutron fields for which the actual neutron energy is near, or even below, the threshold of detection, whilst the NTA film will be calibrated with radioactive neutron sources. The energy response of a dosemeter containing an NTA film has recently been investigated by the National Radiological Protection Board (NRPB) [4]. The neutron response found for $\mathrm{AmBe}$ and ${ }^{252} \mathrm{Cf}$ sources in Ref. 4 has been fitted into the curve drawn through the points for monoenergetic neutrons (Fig. 2).

In stray neutron fields around the high-energy proton accelerators at CERN, the response of the NTA film badge has been found to be up to 35 tracks per $\mathrm{mm}^{2}$ and $\mathrm{mSv}-\mathrm{a}$ value which corresponds to a typical neutron spectrum with energies even higher than $14.7 \mathrm{MeV}$. In order to estimate the dose equivalent from the track density in the emulsion, the neutron energy range in the stray neutron field has to be known. In practice, the variation in response is of the order of a factor of 2. This may be improved, however, by an analysis of the track length distribution using the irradiated emulsion with the help of films which have been exposed to monoenergetic neutrons.

\subsection{Angular response}

The angular response presented in Table 1 has been investigated for film badges containing the NTA film and for irradiations free in air as well as at the front face of an elliptical phantom [6]. Irradiations free in air show a lower track density owing to the absence of fast neutrons back-scattered from the body or phantom. Later experiments have confirmed the earlier results. The various thicknesses of the packing material in front and at the back of the nuclear emulsion result in a different angular response for neutron energies higher than $5 \mathrm{MeV}$. If the unshielded NTA film is irradiated from the front face or the back face, a factor of 1.4 for $14 \mathrm{MeV}$ neutrons is found (see also Fig. 1). 


\subsection{Fading}

All photographic emulsions are subject to fading, i.e. the loss of latent information in the period between the exposure and the development of the film emulsion. Fading is particularly important at high humidities and temperatures. The presence of oxygen plays a decisive role, the loss of latent information being explained by the following chemical reaction: $2 \mathrm{Ag}+\mathrm{O}+\mathrm{H}_{2} \mathrm{O} \rightarrow$ $2 \mathrm{Ag}^{+}+2 \mathrm{OH}^{-}$, i.e. silver atoms formed by the interaction of light or ionizing radiation are re-oxidized to silver ions.

Fading is considered to be the greatest drawback of nuclear emulsions. At room temperature and $100 \%$ relative humidity (R.H.) all latent tracks disappear after only three days. Many investigations have been performed on the influence of temperature and relative humidity on the fading of nuclear tracks. Whilst the general trend in all those observations was the same, not all experiments gave the same result on the absolute scale, as too many parameters had to be controlled. The interested reader should consult the literature for numerical details of earlier experiments [7-9].

From all these investigations the following conclusion can be drawn. Whilst fading is moderate at room temperatures and up to $50 \%$ (R.H.), it increases rapidly at higher temperatures and higher humidity. If NTA film is dried before use, it takes from one to two days until equilibrium with the environment is reached. The normal wrapping paper around the film thus serves as a barrier towards the penetration of humidity. Sealing the dried film into an aluminized plastic pouch prior to use may reduce the fading considerably. A further improvement can be achieved by performing the operation inside a glove box in an atmosphere of dry nitrogen. The drying process should, however, be done carefully, as the emulsion starts to crack and could even become detached from its plastic support. Extreme dehydration also seems to lead to a slight desensitization of the emulsion [10]. Furthermore, electric discharges could occur while unwrapping the film in the darkroom.

Fading is also dependent on the quality of track recording. It is more pronounced for short tracks or low neutron energies, because the loss of a few grains not being developed no longer allows a short track to be distinguished from the background graininess of the emulsion apparent in the microscope image. For long and thin tracks formed at higher neutron energies, only a few thin grains constitute the trace of the recoil proton. Also, under these circumstances the fading effect is more pronounced [11,12].

Various studies have shown that the fading can be largely reduced when the conditions of development are optimized. By using more aggressive developers or by prolonging the development time, the background is still tolerable, and latent silver grains of tracks which, because of fading, are no longer developed under normal conditions, become visible again [11].

Figure 3 shows results of a recent experiment on track fading in the NTA emulsion after irradiation with ${ }^{252} \mathrm{Cf}$ neutrons [13]. The films were not sealed, but were treated in a developing machine by a strongly reducing developer at $29^{\circ} \mathrm{C}$. At room temperature and $50 \%$ R.H., fading after one month is reduced to only $20 \%$, whilst at the temperature of the human body, with the same relative humidity, the number of tracks which can be counted is reduced to one tenth for the same period. At room temperature and $90 \%$ R.H. as well as at $50{ }^{\circ} \mathrm{C}$ and $50 \%$ R.H., tracks disappeared already after three days. In Fig. 4 it is shown that for sealed NTA emulsion at $20^{\circ} \mathrm{C}$ and about $20 \%$ R.H. and for high-energy neutrons, fading can be reduced to $20 \%$ over the usual period of one month. The rapid fading during the first day after irradiation [10] is, however, irrelevant for any calibration and application, as several days usually elapse before film badges are delivered for processing. 
The best results also at higher temperatures are obtained when films are dried and sealed under nitrogen, whilst the storage of the sealed package at $35^{\circ} \mathrm{C}$ and up to $93 \%$ R.H. showed almost no fading after 16 months [14].

Sealing large quantities of NTA emulsion inside a glove box in a dry nitrogen atmosphere is a cumbersome task. The second best choice would be to seal dried films in air, which does not completely avoid fading but at least assures a reproducibility of the results under various atmospheric conditions. The small degree of fading has to be taken into account when considering the over-all uncertainty of the track-counting technique.

\subsection{Dose range}

By scanning a field of about $1 \mathrm{~mm}^{2}$ on a nuclear emulsion, a lower dose limit of $0.4 \mathrm{mSv}$ can be reached. As an upper limit, a dose equivalent of $200 \mathrm{mSv}$ is usually quoted. This is true for neutrons in the optimum detection range between 2 and $4 \mathrm{MeV}$. At higher energies, long tracks will cross each other and considerably blur the picture. The distinction of single tracks becomes more and more difficult with higher doses, so that for neutrons in stray fields of high-energy proton accelerators an upper limit of $50 \mathrm{mSv}$ is a reasonable figure.

\subsection{Gamma sensitivity}

The limits on dose range for neutrons are highly affected if NTA emulsions are exposed in addition with gamma radiation. The gamma background develops in the form of black grains of silver in the emulsion and makes the distinction of tracks more and more difficult. Problems of counting start at an irradiation with $10 \mathrm{mSv}$ of gamma radiation and $0.5 \mathrm{mSv}$ of soft-X radiation. In the case of an exposure with hard-photon radiation of $20 \mathrm{mSv}$ or the corresponding figure at lower energies, track recognition becomes almost impossible.

A lead filter in the front part of the badge may reduce the fogging of the emulsion from a photon exposure, but it also prevents recoil protons produced in the plastic holder from reaching the emulsion [4].

\subsection{Sensitivity to thermal neutrons}

The sensitivity of the NTA film to thermal neutrons due to the ${ }^{14} \mathrm{~N}(\mathrm{n}, \mathrm{p}){ }^{14} \mathrm{C}$ reaction disturbs the detection of fast neutrons. Compared to Am-Be neutrons, the response to thermal neutrons has been found to be $45 \%$ higher. Thermal neutrons can be discriminated by a cadmium filter around the film. A similar response to thermal and fast neutrons can be achieved by placing a boron-loaded plastic filter at the back of the film holder to absorb the albedo thermal neutrons emitted from the body.

\section{UNCERTAINTY OF DOSE MEASUREMENT}

\subsection{Background track distribution}

Background tracks in nuclear emulsions are generally not a problem. Independently of the storage time, a background of somewhat more than 1 track per $\mathrm{mm}^{2}$ is reported. The natural fading of unsealed film in a normal atmosphere obviously tends to stabilize the above figure. Extensive measurements have shown that the frequency of background tracks follows closely a binomial distribution as shown in Fig. 5 [10]. 


\subsection{Over-all uncertainty}

The uncertainty of dose measurement is not only affected by the absolute number of background tracks and its variation within the batch; the emulsion as a detector shows some variation in sensitivity even for films of the same production batch. Experiments showed that the standard deviation due to the influence of the batch uniformity, the development of the film, and the individual track-counting statistics of the order of $10 \%$ [11]. For low doses, the over-all uncertainty increases owing to the counting statistics and the presence of background tracks.

On the basis of experimental data, the standard deviation of the NTA film is plotted in Fig. 6 as a function of neutron dose equivalent following a method described in Ref. [15]. This data for radioactive neutron sources also takes into account effects of fading of $50 \%$ or $10 \%$ on the relative standard deviation. The somewhat higher sensitivity of the NTA emulsion to stray neutrons from high-energy proton accelerators means that a dose of $1 \mathrm{mSv}$ can be determined with a standard deviation of $20 \%$, provided the fading can be controlled to $10 \%$ over the normal wearing period.

\section{TECHNIQUE OF TRACK COUNTING}

\subsection{Visual counting by microscope}

The best technique with respect to counting efficiency - but the worst with respect to time required - is visual counting by microscope. The contrast is greatly enhanced and track structure best analysed by using oil immersion, resulting in a total magnification of $\times 1000$. Such a technique is only used if track recognition is difficult, for instance because of appreciable gamma fogging. In routine microscopical scanning, however, modern objectives ranging from $\times 45$ to $\times 63$ and with high numerical aperture binoculars with magnification $\times 10$ are generally employed. Because NTA films are never completely flat, the focalizing has to be reoptimized for each field if no covering glass is used or if the detector is clamped on its side without any glass support.

The microscopical scanning of the nuclear emulsion, field by field, is a tedious job. It has been said that a person can attentively scan only 20 to 40 films per day without overstraining the eyes or running into problems of overlooked information. As mentioned above, it is not always necessary to scan the full standard area in order to make a decision on a zero exposure. Nevertheless, the direct observation of tracks in a microscope should be reserved for special cases, and other faster techniques should be employed in routine work.

\subsection{Projection method}

In order to avoid the direct observation of tracks in the microscope, the image can be projected onto a screen by means of a prism giving total magnifications of the same order of magnitude as mentioned above. Although a high-pressure mercury lamp is used to improve luminosity, the picture has to be viewed in a dark room. In order to speed up the scanning process, the NTA film is fixed on a tilted motorized table, where a strip corresponding to the area of the emulsion to be scanned is moved through the focal spot of the objective. Owing to the low luminosity and the moving picture, the recognition of tracks is greatly compromised. Experience has shown that compared to a direct observation in a microscope using the discrete field technique, up to half the information is lost, especially for tracks which have already been subject to some fading. 


\subsection{Television camera and monitor}

By placing a television camera instead of binoculars on top of a microscope it becomes possible to transmit the video signal to a monitor. Whilst a simple camera and a cheap black-and-white monitor already give satisfactory results, further improvement can be made by carefully selecting the components. One-inch camera tubes are preferable to smaller sizes. The camera should be of good quality, the image being sharp even in the corners. Monitors should allow an enhancement of contrast beyond the one usually found in safeguard or surveying equipment.

An objective of $\times 25$ is usually sufficient for observing clear tracks on the television screen and for limiting routine scanning to two fields. If no track is found in two fields, then there is only a $1 \%$ probability of missing a dose of $0.5 \mathrm{mSv}$, whilst $1 \mathrm{mSv}$ is detected with certainty. If one track has been counted in two fields, it is recommended to scan another three fields, bringing the total area to the normal size of about $1 \mathrm{~mm}^{2}$. This technique allows a great number of zero-dose films to be scanned in a short time. In the rare case of doses higher than $1 \mathrm{mSv}$, it is recommended that observation by television cameras and monitors be complemented by a special scanning in the microscope.

\subsection{Automatic counting}

In spite of the considerable efforts that have been made to automize the scanning of neutron films, no system is used in a routine way. There are three methods of automatic scanning: mechanical, electronic, and flying-spot scanning. The latest developments in this field are presented in Ref. [16]. The major drawback of automatic scanning devices is, apart from the price, the reduced detection efficiency compared with direct microscopical observation. These figures range from $50 \%$ to $70 \%$, while at the same time a higher background is found in non-irradiated emulsions due to bad discrimination of the automatic devices against pseudotracks, e.g. scratches.

\section{PRACTICAL ASPECTS OF ROUTINE MONITORING}

It has already been mentioned that the conversion of counted tracks to dose equivalent is performed using a standard calibration factor that has to be determined for the installation of interest: for instance, by field calibration, and taking into account all factors such as development conditions, fading behaviour, and counting techniques. This is acceptable as long as recorded doses are not too high (below $1 \mathrm{mSv}$ ). For higher doses a closer analysis of the results becomes necessary. Further analysis of the recorded tracks has to be applied, especially when the distribution in track length of the actual film is different from that found for the typical spectrum at the installation [10].

A serious underestimation of dose equivalent would result in cases where the person wearing his dosemeter in front was irradiated from the back. Personnel exposures in neutron stray fields are, however, more frequent than beam irradiations. Under the usual circumstances of a quasi-isotropic radiation field, the decision whether to use the front-face calibration or to consider the rotation of the phantom becomes somewhat irrelevant if again field calibrations are used to establish the actual response of the NTA film.

General experience shows, however, that in the majority of installations neutron exposures are very low. Most of the films evaluated monthly show background tracks only, so that the NTA film serves as a monitor rather than as a dosemeter, ensuring that no neutron exposure has occurred. In 
this case it is a time-consuming job to apply the full scanning procedure over the complete standard area. Therefore only part of the total area should be scanned in order to be sure that no appreciable personnel dose has occurred. Only if tracks are found during such a quick scanning procedure should more fields be counted until the desired statistical uncertainty is reached.

On the other hand, it is not necessary to count more than about 100 tracks in order to decrease the counting statistics. It has been mentioned above that the over-all uncertainty of track counting cannot be better than $10 \%$ (see also Fig. 6) even when the stochastic uncertainty has become extremely small by counting a great number of tracks. For high track densities, only part of the standard area must be scanned.

It has been shown that for a given dose the expected number of tracks can be described by a binomial probability distribution. At the lower detection limit it is useful to report personnel doses in classes with steps of $0.2 \mathrm{mSv}$. Thus the number of counted tracks will be converted to doses and sorted into classes, with a cumulative probability above $50 \%$. According to this approach, two tracks above the usual background of $1 \pm 1$ track (in a standard field of $1 \mathrm{~mm}^{2}$ ) are still considered to be zero dose rather than belonging to the lowest class of $0.2 \mathrm{mSv}$.

The recommended techniques of quick scanning in areas of different size and of dose classification recently discussed in detail [10] could make an appreciable saving in manpower when a great number of dosemeters have to be treated.

\section{CONCLUSIONS}

The NTA film is still widely used as a personnel dosemeter in stray radiation fields of neutron energies below $1 \mathrm{MeV}$ where, even in the case of high exposures, no tracks are recorded. This calls for replacement of the nuclear emulsion by other neutron detectors which are sensitive to low-energy neutrons.

Only some dosemeter services make use of sealing techniques and have taken measures to avoid, or at least to reduce, the known fading of the nuclear emulsion. Variations in the great number of parameters which may affect the conditions of sealing and development of the nuclear emulsion prevented these techniques (from being standardized) and also the transfer of fading results from one laboratory to another. Hence, it is not astonishing that the nuclear emulsion has a bad reputation as a personnel dosemeter.

If, when using NTA film, the necessary precautions are taken to avoid fading, it should be possible to get dosimetric information in radiation fields containing neutron components well above $1 \mathrm{MeV}$. For a conversion of nuclear tracks into dose equivalent, a calibration should preferably be performed in the radiation field in which the dosemeter is worn. 


\section{REFERENCES}

[1] H. Kiefer and R. Maushart, Radiation protection measurements (Pergamon Press, Oxford, 1972).

[2] K. Becker, Solid-state dosimetry (Cleveland CRC-Press, 1973).

[3] R.A. Dudley, Dosimetry with photographic emulsion in Radiation Dosimetry, vol. II (Academic Press, Inc., New York, 1966), p. 326.

[4] D.T. Bartlett, T.V. Bird and J.C.H. Miles, The NRPB nuclear emulsion dosemeter, NRPB Report No. 99 (1980).

[5] J.S. Cheka, Recent developments in film monitoring of fast neutrons, Nucleonics 12 (6), 40 (1954).

[6] E. Piesch, Zur Dosimetrie schneller Neutronen mit Kernspurfilmen, Atompraxis 9, H5, 179 (1963).

[7] K. Becker, Fehlerquellen bei der Neutronen-Personendosismessung mittels Kernspuremulsionen, Atomkernenergie 8, 74 (1963).

[8] A.M. Sayed and E. Piesch, Study of the latent fading of NTA film and track-etch detectors, KFK 2032(1974).

[9] P.N. Krishnamoorthy, Comparison of various neutron personnel dosimeters, Proc. IAEA, Symp. on Neutron Monitoring and Radiation Protection, Vienna, 1972 (IAEA, Vienna, 1973), Vol. II, p. 343.

[10] M. Höfert, The NTA emulsion: An ill-reputed but misjudged neutron detector, Proc. 10th DOE Workshop on Personnel Neutron Dosimetry, Acapulco, 1983 (to be published).

[11] J. David, M. Höfert and K.H. Ritzenhoff, New results on the detection of neutrons by track emulsions, Proc. 16th Annual Meeting of Fachverband für Strahlenschutz, Munich, 1982 (Strahlenschutzmesstechnik, GSF Bericht FS-83-30-T, p. 189, June 1983).

[12] D.T. Bartlett, A. Knight and T.O. Marchal, A study of the NRPB fast neutron personal monitoring service, NRPB Report NRPB-R-40 (1975).

[13] K.H. Ritzenhoff and B. Burgkhardt, private communication, 1983.

[14] D.T. Bartlett and F.L. Creasey, Latent image fading in nuclear emulsions, Phys. Med. Biol. 22(5), 733 (1977).

[15] E. Piesch, J. Jasiak and M. Urban, Makrofol and CR39 recoil track etch detectors as a supplement of a universal albedo neutron dosimeter, Proc. 12th Int. Conf. on Nuclear Track Detectors, Acapulco, 1983 (to be published).

[16] R. Gold, J.H. Roberts, C.C. Preston, J.P. McNeece and F.H. Ruddy, The status of automated nuclear scanning systems, Proc. 12th Int. Conf. on Nuclear Track Detectors, Acapulco, 1983 (to be published). 
Table 1

Influence of the direction of radiation incidence and of the body on the indication of nuclear film type A (NTA) for PoBe neutrons and for $14 \mathrm{MeV}$ neutrons [6]. The free-air exposure to the front has been normalized to $100 \%$.

\begin{tabular}{|c|c|c|c|c|c|c|c|c|}
\hline & \multicolumn{4}{|c|}{ Phantom exposure (\%) } & \multicolumn{4}{|c|}{ Free-air exposure (\%) } \\
\hline & $\begin{array}{l}\text { Front } \\
\rightarrow\end{array}$ & $\begin{array}{l}\text { Back } \\
\rightarrow\end{array}$ & $\begin{array}{r}\text { Side } \\
\rightarrow\end{array}$ & $\begin{array}{l}\text { Rotating } \\
\rightarrow \text { : }\end{array}$ & Front & Back & Side & Rotating \\
\hline $\begin{array}{l}\text { PoBe } \\
\text { neutrons }\end{array}$ & $\begin{array}{l}132.5 \\
\pm 5.7\end{array}$ & $\begin{array}{l}18.7 \\
\pm 8.7\end{array}$ & $\begin{array}{l}65.6 \\
\pm 5.2\end{array}$ & 65.5 & 100 & $\begin{array}{l}100 \\
\pm 6\end{array}$ & $\begin{array}{l}52.7 \\
\pm 5.9\end{array}$ & 76.4 \\
\hline $\begin{array}{l}14 \mathrm{MeV} \\
\text { neutrons }\end{array}$ & $\begin{array}{r}108 \\
\pm 6\end{array}$ & $\begin{array}{c}58 \\
\pm 7.3\end{array}$ & $\begin{array}{l}76.6 \\
\pm 6.9\end{array}$ & 76.3 & 100 & $\begin{array}{c}139 \\
\pm 4.6\end{array}$ & $\begin{array}{c}69 \\
\pm 5.4\end{array}$ & 94.5 \\
\hline
\end{tabular}




\section{Figure captions}

Fig. 1 Calculated recoil proton yield of a) a Kodak NTA nuclear film, and b) a film combination shielded by a $57 \mathrm{mg} / \mathrm{cm}^{2}$ aluminium foil and a plastic radiator of $116 \mathrm{mg} / \mathrm{cm}^{2}$ thickness as a function of neutron energy [6]. Film and combination were irradiated from the front (- $\longrightarrow$ and the back (----).

Fig. 2 Normalized dose equivalent response of NTA emulsion as a function of neutron energy in $\mathrm{MeV}$ as measured by Ref. [4].

Fig. 3 Fading of nuclear tracks from source neutrons as a function of storage time after irradiation. The parameter in the upper figure is the temperature; that in the lower one is the relative humidity (R.H.). Films were developed in a strongly reducing developer at $29^{\circ} \mathrm{C}$.

Fig. 4 Fading of nuclear tracks from high-energy neutrons at room temperature as a function of storage time after irradiation. Films were dried and sealed before use.

Fig. 5 Measured background distribution of tracks in NTA emulsion and fitted binomial distribution function $P_{B}(N, 3,0.352)$ for a field size of $0.875 \mathrm{~mm}^{2}$. The mean value is 1.056 tracks, the standard deviation is 0.873 .

Fig. 6 Relative standard deviation in percent as a function of dose equivalent in $\mathrm{mSv}$ when scanning a surface of $1 \mathrm{~mm}^{2}$. Curves are calculated for $\mathrm{PuBe}$ source neutrons for $10 \%$ and for $50 \%$ fading, as well as for high-energy neutrons ( $10 \%$ fading). 


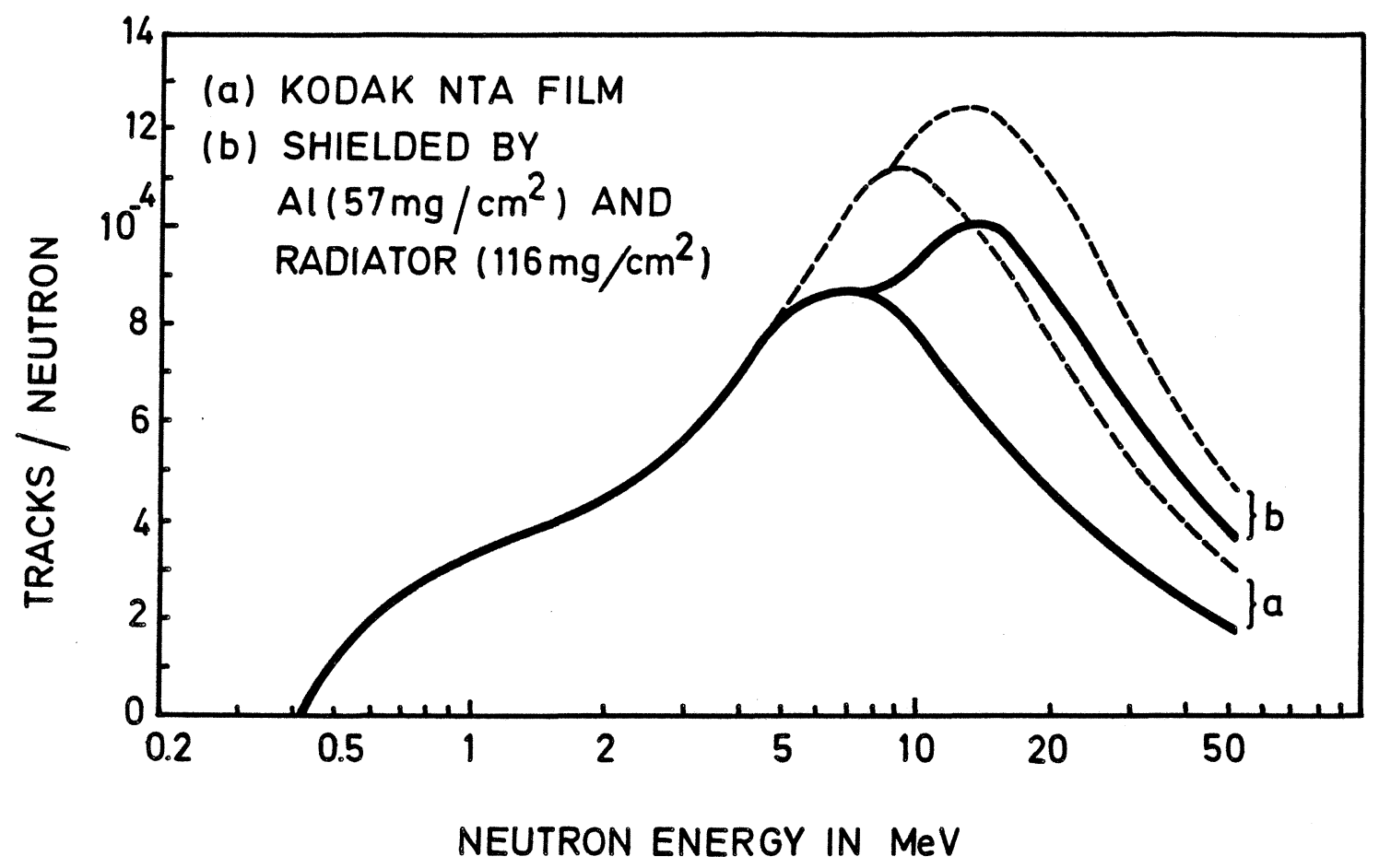

Fig. 1

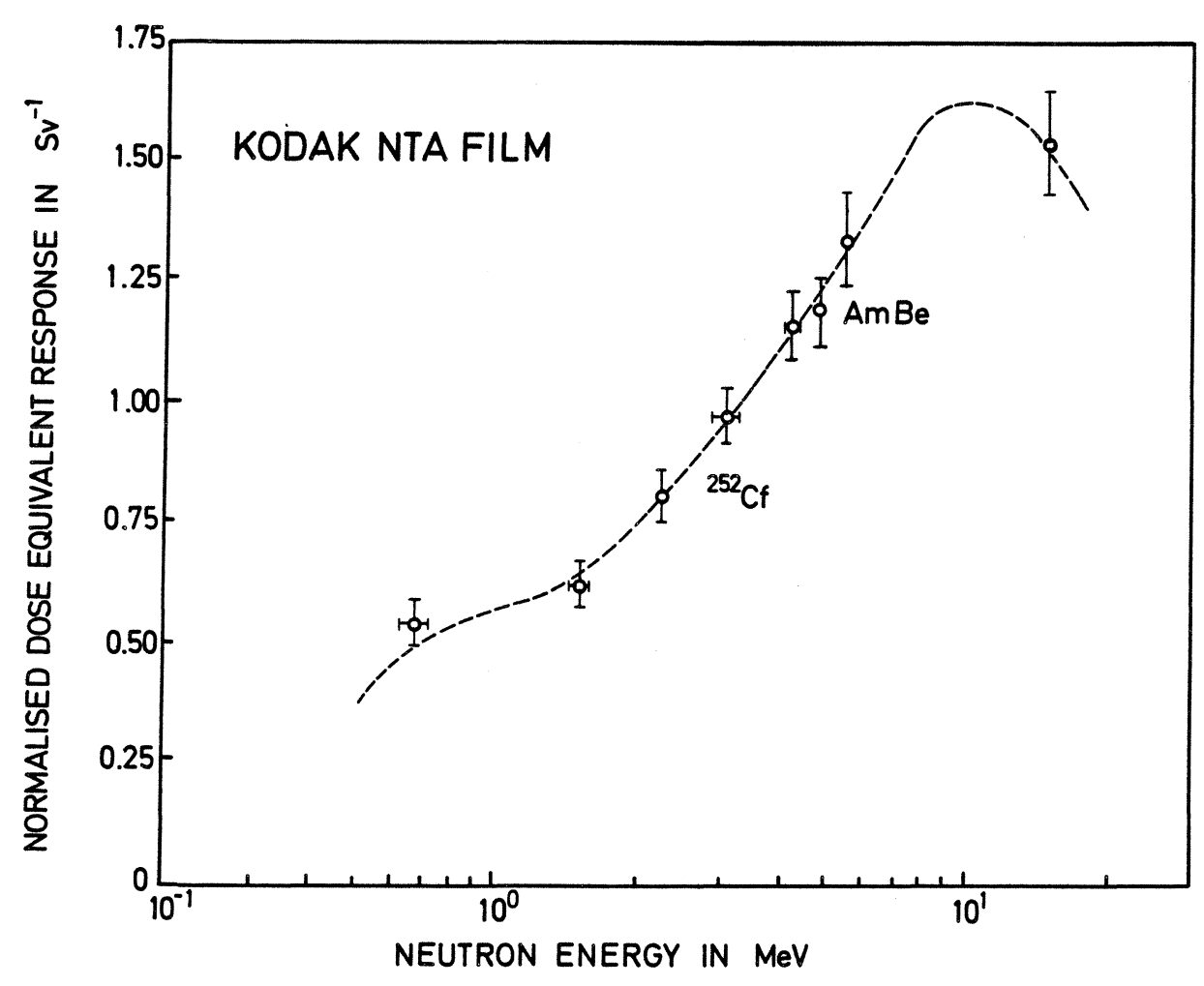

Fig. 2 

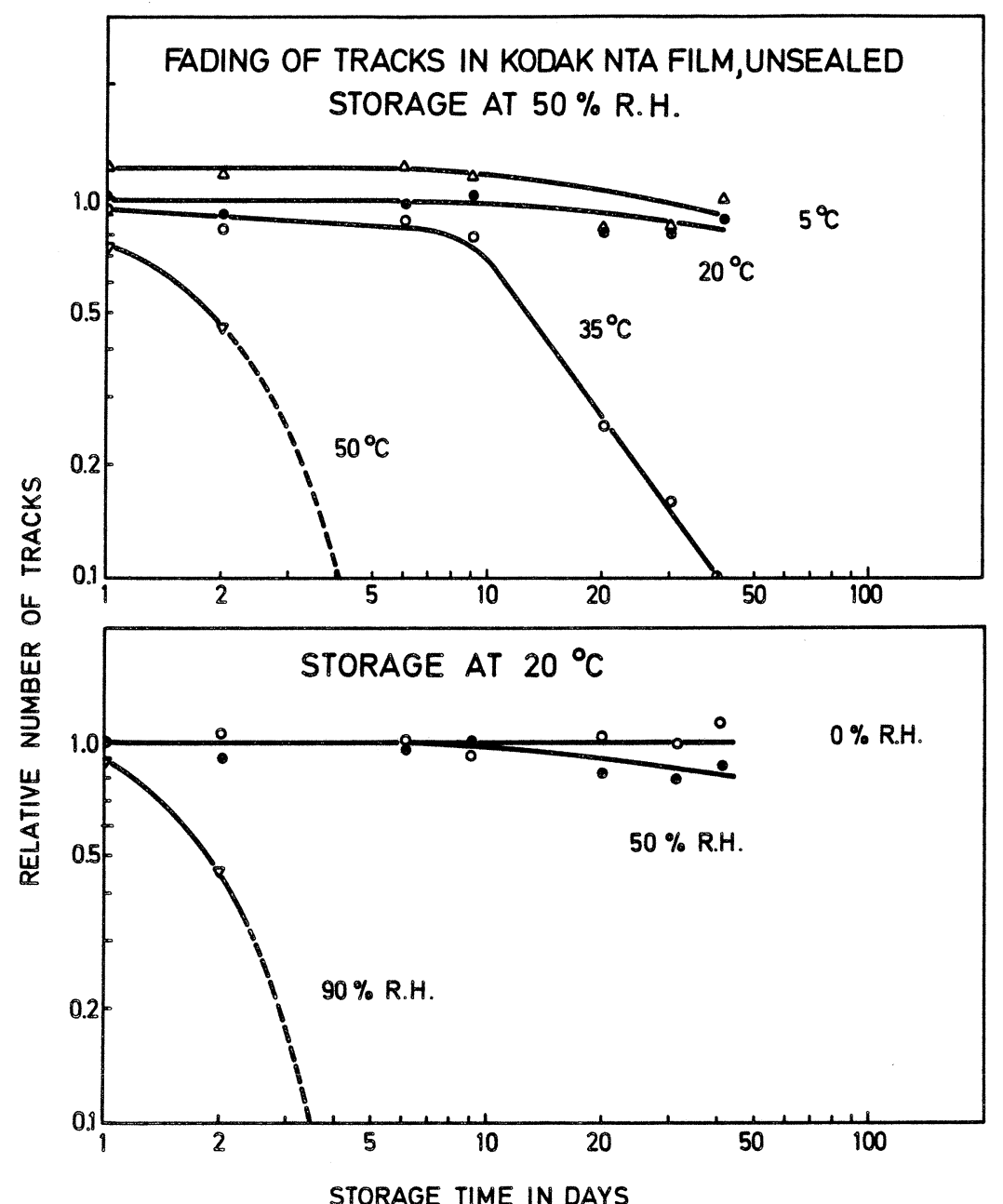

Fig. 3

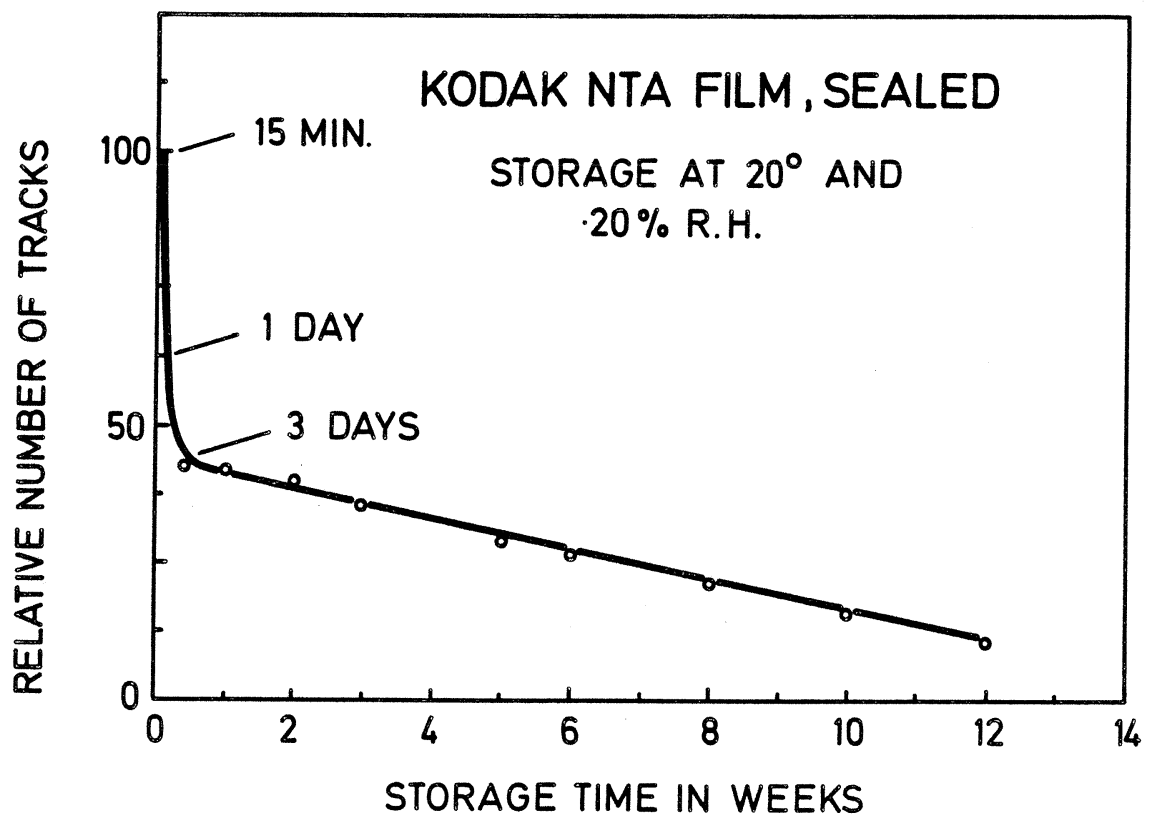

Fig. 4 


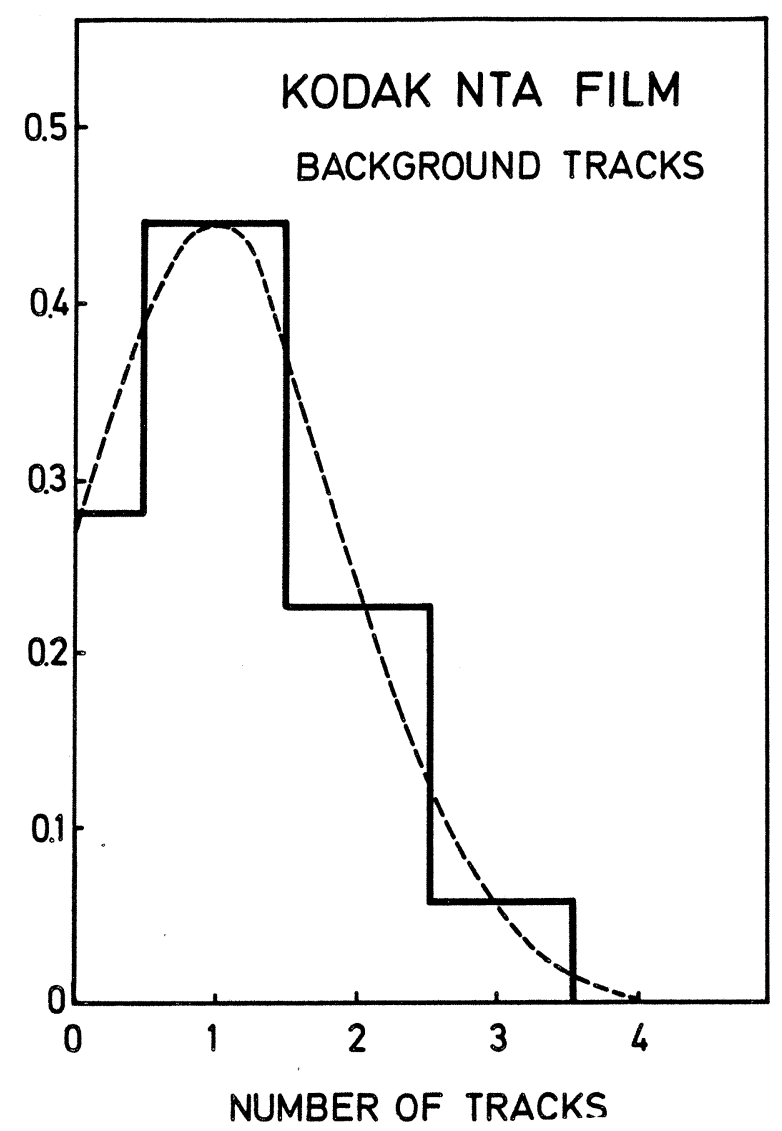

Fig. 5

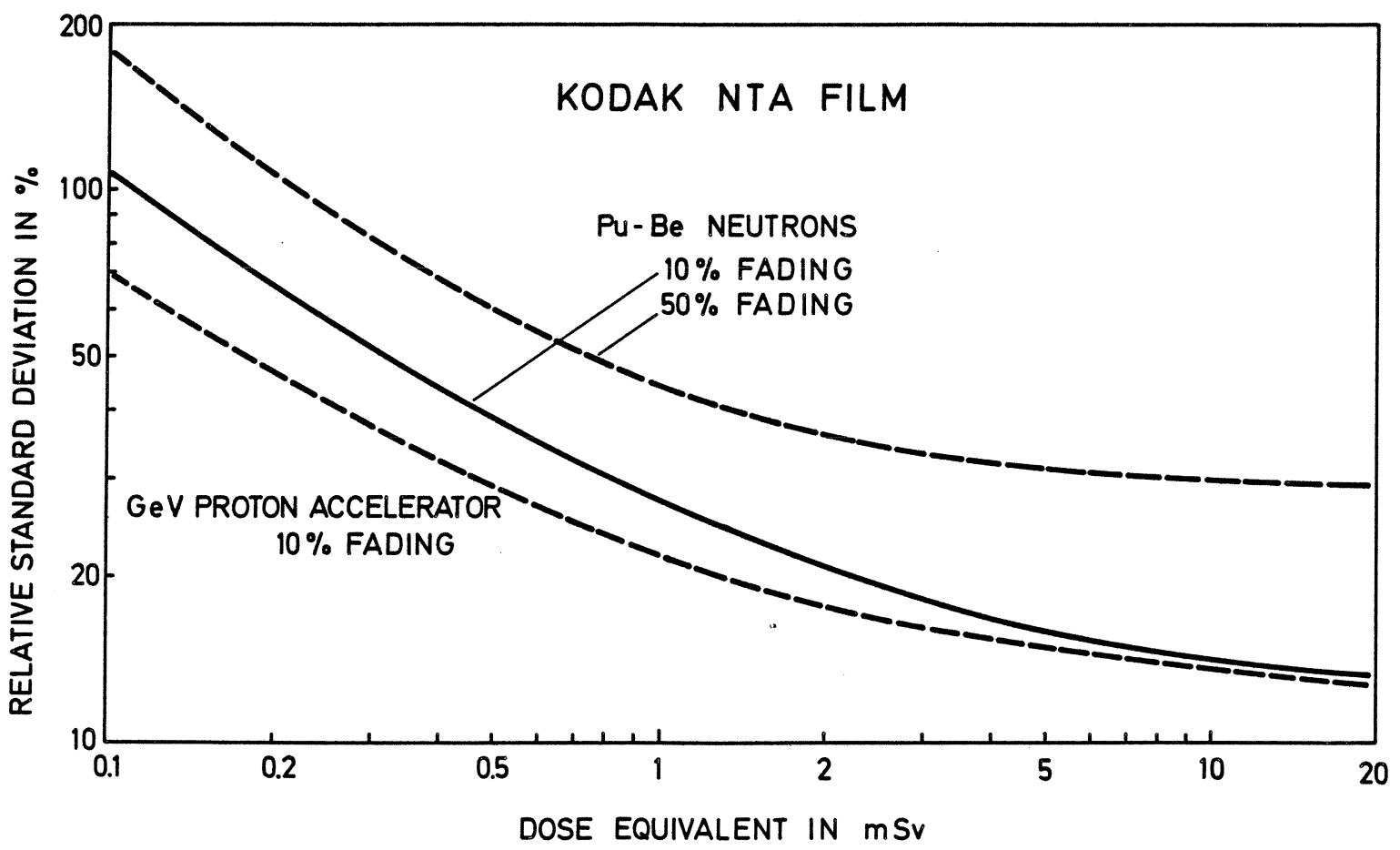

Fig. 6 